\title{
Originalien
}

Notfall Rettungsmed 2022 $25: 177-185$ https://doi.org/10.1007/s10049-020-00835-z Angenommen: 15. Dezember 2020

Online publiziert: 15. Januar 2021

(c) Springer Medizin Verlag $\mathrm{GmbH}$, ein Teil von Springer Nature 2021

Julian Ganter $r^{1,2,3} \cdot$ Domagoj Damjanovic ${ }^{1,2,3} \cdot$ Georg Trummer ${ }^{1,2,3} \cdot$ HansJörg Busch ${ }^{2,3,4}$. Klemens Baldas ${ }^{2,3,5}$. Thomas Steuber ${ }^{2}$. Jan Niechoj ${ }^{2,6}$. Michael P. Müller ${ }^{2,3,5}$

' Klinik für Herz- und Gefäßchirurgie, Universitäts-Herzzentrum Freiburg - Bad Krozingen, Medizinische Fakultät, Albert-Ludwigs-Universität Freiburg, Freiburg, Deutschland

${ }^{2}$ c/o Klinik für Anästhesiologie, Intensiv- und Notfallmedizin, St. Josefskrankenhaus, Region der Lebensretter e. V., Freiburg, Deutschland

${ }^{3}$ Deutscher Rat für Wiederbelebung e. V. - German Resuscitation Council, Ulm, Deutschland

${ }^{4}$ Universitäts-Notfallzentrum, Universitätsklinikum Freiburg, Freiburg, Deutschland

${ }^{5}$ Klinik f. Anästhesiologie, Intensiv- und Notfallmedizin, St. Josefskrankenhaus, Freiburg, Deutschland

${ }^{6}$ Kreisverband Freiburg e. V., Deutsches Rotes Kreuz, Freiburg, Deutschland

\section{Implementierungsprozess einer Smartphone-basierten Ersthelferalarmierung}

\section{Herausforderungen bei der Einführung, Weiterentwicklung zum System 2.0}

Rettungsdienst und den Leitstellen nicht $\mathrm{zu}$ erreichen [4].

In der deutschen Bevölkerung verwenden $70 \%$ der Bürger ein Smartphone [8], darunter viele Personen mit medizinischer Qualifikation. Es ist naheliegend, bei einem Herz-Kreislauf-Stillstand Personen zu aktivieren, die sich in unmittelbarer Nähe befinden. Ringh zeigte, dass die Ortung von Ersthelfern über Mobilfunkmasten und Alarmierung über Kurznachricht (SMS) das therapiefreie Intervall um mehrere Minuten reduzieren können [11]. Seit 2012 ist in Dänemark das System FirstAED etabliert. Dieses ortet Ersthelfer über eine Smartphone-App, alarmiert gleich mehrere Helfer und teilt Aufgaben zu. Ein bis zwei Helfer werden zum Patienten geschickt, ein weiterer wird zum nächstgelegenen AED geschickt und bringt diesen zum Notfallort [6].

In Deutschland sind verschiedene App-basierte Ersthelferalarmierungssysteme etabliert. Eine Erhebung hierzu bei deutschen Rettungsleitstellen wurde 2018 von Gross veröffentlicht und dokumentierte dabei auch typische und wiederkehrende Hürden und Herausforderungen bei der Etablierung [5].
In Baden-Württemberg startete 2016 ein Pilotprojekt, in dem unter Koordination des Ministeriums für Inneres, Digitalisierung und Migration in drei verschiedenen Landkreisen die damals verfügbaren Ersthelferalarmierungssysteme (corhelp 3r, FirstAED, Mobile Retter) etabliert und evaluiert werden sollten [10]. Mittlerweile sind mindestens zwei weitere Ersthelferalarmierungssysteme in Deutschland kommerziell verfügbar.

Zusätzlich zur Alarmierung von Ersthelfern kann ein ganzheitliches Herangehen an das Problem des außerklinischen Herz-Kreislauf-Stillstands sinnvoll sein. Im Tessin wurde ein beeindruckendes Netzwerk öffentlich zugänglicher AED geschaffen, die alarmierten Ersthelfer sehen die verfügbaren AED in ihrer App [1]. In der Region Süddänemark ist die Alarmierungs-App FirstAED ebenso an eine AED-Datenbank angebunden. Von den drei alarmierten Helfern wird einer von der App zum nächsten AED geleitet [13].

Ziel des Projekts war die Etablierung und Evaluation eines Smartphone-basierten Ersthelferalarmierungssystems sowie die Weiterentwicklung und Anpassung an die regionalen Strukturen. 


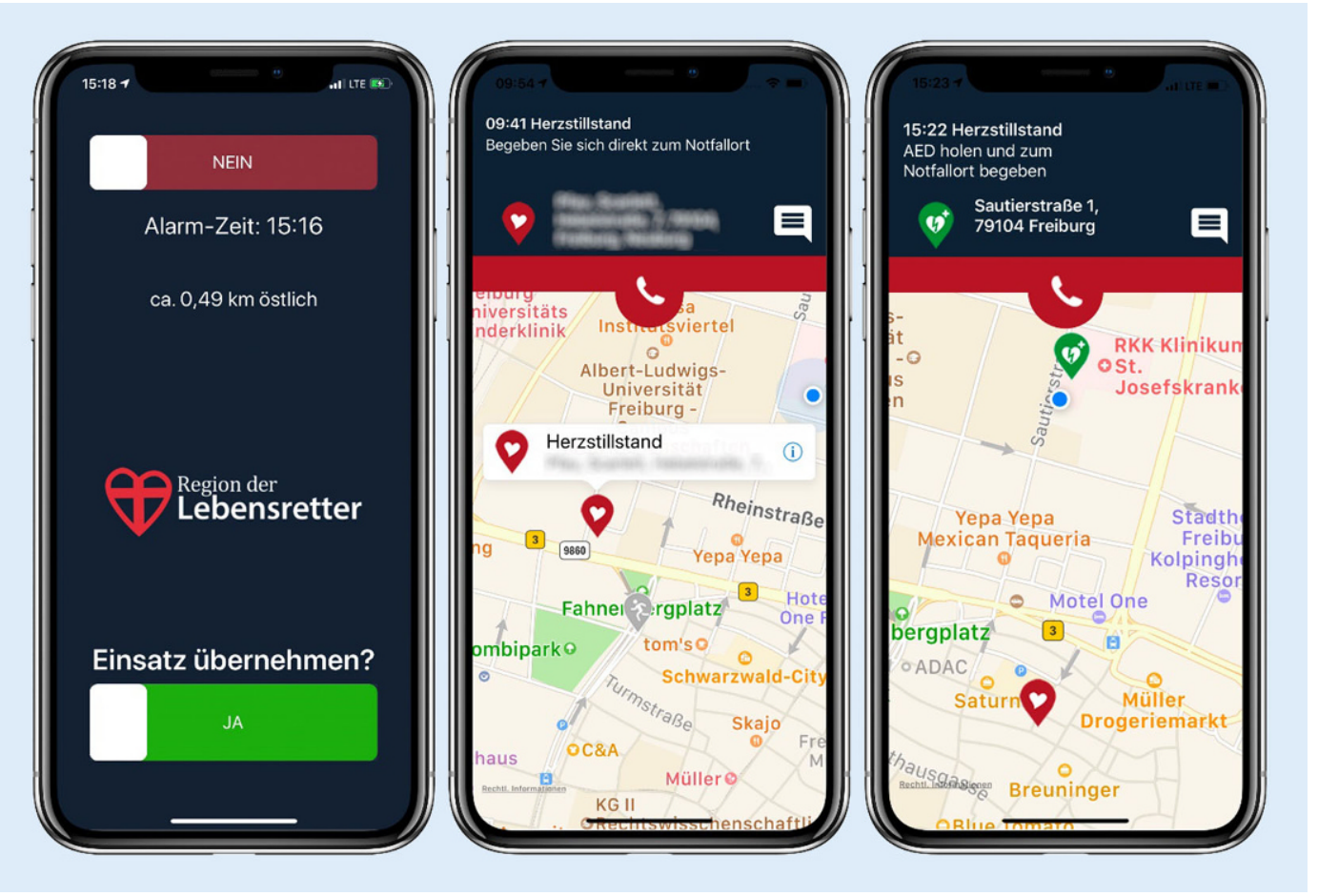

Abb. $1<1$. Alarmdisplay es werden die Alarmzeit und die Entfernung zum Notfallort angezeigt. 2. Anzeige auf dem Smartphone des Helfers, der direkt zum Notfallort geschickt wird. 3. Anzeige auf dem Smartphone des Helfers, der den AED holen soll. Die Textbox oben rechts enthält weitere Angaben der Leitstelle. $(\odot$ all rights reserved, mit freundlicherGenehmigung von FirstAED)
Im Fokus der Evaluation stand die Evaluation der Ersthelferzahlen, der Ersthelferverfügbarkeit bei Alarmierungen, der Erfassung der Eintreffzeiten sowie der Verfügbarkeit von AED bei der Versorgung durch die Ersthelfer.

\section{Studiendesign und Untersuchungsmethode}

\section{Projektregion Freiburg}

Das System wurde im Bereich der Integrierten Leitstelle (ILS) Freiburg etabliert. Dazu zählen die Stadt Freiburg (230.241 Einwohner, $153 \mathrm{~km}^{2}$ ) sowie der Landkreis Breisgau-Hochschwarzwald (262.795 Einwohner, $1378 \mathrm{~km}^{2}$ ).

\section{System FirstAED}

FirstAED besteht aus einer ErsthelferApp sowie einer Server-Software, die innerhalb der Leitstellen-Firewall installiert wird. Die Anbindung an das Einsatzleitsystem (Siveillance Command, Siemens, München) wurde über Rescuetrack (Convexis GmbH, Reutlingen) realisiert.

An den FirstAED-Server angebunden ist die App „Region der Lebensretter“ für Smartphones (Android, iOS). Nach
Registrierung und Einweisung erhalten die Ersthelfer ein Login zum Freischalten der App.

\section{Organisation und Struktur}

Für den Betrieb und die Weiterentwicklung der Software, die Registrierung der Helfer und die Finanzierung des Projekts wurde der Verein Region der Lebensretter e. V. gegründet, der Verein ist korporatives Mitglied im DRK Kreisverband Freiburg e. V.

Bei einer Alarmierung mit den Einsatzstichworten „bewusstlos“ und „Reanimation“ werden die Ersthelfer automatisch über ihr Smartphone geortet und alarmiert, sofern sie sich in einem definierten Radius um den Einsatzort befinden (• Abb. 1). Medizinische Einrichtungen (z. B. Pflegeheime) als Einsatzort sind von der Alarmierung ausgeschlossen. Von den Ersthelfern, die den Einsatz annehmen, erhalten zwei die Aufgabe, sich zum Notfallort zu begeben und die Basisreanimation einzuleiten, ein Helfer wird zum nächstgelegenen AED geleitet. Der vierte Ersthelfer weist den Rettungsdienst ein bzw. betreut die Angehörigen. Sobald der Einsatz vom Helfer angenommen wird, erscheint dieser als Rettungsmittel auf der Kartenübersicht der ILS.
Hilfe nach besonders belastenden Einsätzen wird den Helfern beim Ausfüllen des Helferberichts angeboten und über die Vermittlung zur psychosozialen Notfallversorgung (PSNV) organisiert.

Bereits vorhandene Helfer-vorOrt(HvO)-Systeme bleiben unverändert in der Alarm- und Ausrückeordnung (AAO) berücksichtigt. Die HvO-Gruppen wurden eingeladen, sich im Projekt $\mathrm{zu}$ registrieren.

\section{Ersthelfer}

Mindestqualifikation ist eine Sanitätshelferausbildung. Pflegekräfte und Ärzte sind qualifiziert, sofern sie regelmäßig die Maßnahmen des Basic Life Support trainieren.

In den Hilfsorganisationen, Feuerwehren und Krankenhäusern in der Region gibt es verantwortliche Personen, die Helfer registrieren. Jeder Helfer wird mit Warnweste, Taschenbeatmungsmaske und Einmalhandschuhen ausgestattet.

\section{Öffentlich zugängliche automatisierte externe Defibrillatoren (AED)}

Durch eine strukturierte Recherche wurden AED-Standorte eruiert und in die 
Notfall Rettungsmed 2022 ·25:177-185 https://doi.org/10.1007/s10049-020-00835-z

(c) Springer Medizin Verlag GmbH, ein Teil von Springer Nature 2021

J. Ganter · D. Damjanovic · G. Trummer · H.-J. Busch · K. Baldas · T. Steuber · J. Niechoj · M. P. Müller

Implementierungsprozess einer Smartphone-basierten Ersthelferalarmierung. Herausforderungen bei der Einführung, Weiterentwicklung zum System $\mathbf{2 . 0}$

\section{Zusammenfassung}

Hintergrund. Die Verkürzung des reanimationsfreien Intervalls beim Herz-KreislaufStillstand erhöht die Überlebensrate. Smartphone-basierte Systeme können Ersthelfer in der Nähe des Notfallorts orten und alarmieren.

Ziel. Etablierung eines Ersthelferalarmierungssystems, technische Weiterentwicklung und Anpassung an regionale Strukturen.

Material und Methoden. Das System „Region der Lebensretter" wurde im Juli 2018 in Freiburg etabliert. Mittels halbjährlicher Evaluation wurde der Bedarf für Optimierungen festgestellt und im Sinne eines PDCA(plando-check-act)-Zyklus umgesetzt. Die nötigen Funktionen wurden spezifiziert („plan“), programmiert, getestet und freigegeben („do $\left.{ }^{\prime \prime}\right)$. Anschließend wurden die Änderungen evaluiert (",check“) und bei Bedarf weitere Optimierungen durchgeführt (,act“). Ergebnisse. Die Zahl der Ersthelfer stieg von 276 (2. Halbjahr 2018) auf 794 Helfer (1. Halbjahr 2020). Die Einsatzübernahmen stiegen von $30 \%$ (2. Halbjahr 2018) bis auf $49 \%$ (1. Halbjahr 2020). Folgende Funktionen wurden programmiert und umgesetzt: dynamischer Alarmierungsradius in Abhängigkeit der voraussichtlichen Eintreffzeit des Rettungsdiensts, lauter Alarm trotz Stummschaltung, Anbindung an AEDDatenbank, Ersthelferausweis, Statusmeldung „eingetroffen", Angabe des Verkehrsmittels zur Optimierung des Algorithmus. Die Anzahl der vorhandenen AED nahm von 190 auf 270 zu.
Diskussion. Smartphone-basierte Alarmierungssysteme können das reanimationsfreie Intervall verkürzen. Neben der Gesamtzahl von Ersthelfern ist die technische Umsetzung entscheidend. Weitere Studien sollten auf der Basis valider Daten untersuchen, ob die Überlebensrate nach außerklinischem HerzKreislauf-Stillstand gesteigert werden kann. Die Anbindung der Systeme an Datenbanken der Qualitätssicherung im Rettungsdienst bzw. Reanimationsregister erscheint sinnvoll.

Schlüsselwörter

Außerklinischer Herz-Kreislauf-Stillstand . App - Reanimation - Reanimationsfreies Intervall · Ersthelfersystem · Automatisierter externer Defibrillator (AED)

\section{Implementation of a smartphone-based first-responder alerting system. Challenges of implementation and development to system $\mathbf{2 . 0}$}

\section{Abstract}

Background. Shortening the resuscitationfree interval in cardiac arrest increases the survival rate. Smartphone-based systems can locate and alert nearby rescuers.

Objectives. Implementation of a first responder system, technical development and adaption to regional structures.

Materials and methods. The system "Region der Lebensretter" was successfully established in July 2018 in Freiburg. The need of optimization was evaluated every half year and realized according to the PDCA (plan-do-check-act) cycle. The necessary functions were specified (plan), programmed, tested and released (do). Afterwards the changes were evaluated (check) and, if necessary, further optimizations were implemented (act).

Results. The number of registered rescuers increased from 276 (2nd half year 2018) to 794 (1st half year 2020). The rate of alarm acceptance increased from $30 \%$ (2nd half year 2018) to $49 \%$ (1st half year 2020). The following features were designed and released: dynamic adjustment of the alarm radius (DAA), critical alert function, connection to automated external defibrillator (AED) database, digital rescuer identification (ID), feedback button "arrived on scene", choice of means of transport for algorithm optimization. The number of existing AEDs increased from 190 to 270.
Conclusion. The resuscitation-free interval can be shortened by smartphone-based alerting systems. For successful operation, the total number of rescuers and the technical realization is crucial. Further studies are necessary to investigate whether the survival rate of out-of-hospital cardiac arrest can be increased. It appears extremely appropriate to adapt these systems to databases of quality management or research registers.

Keywords

Out-of-hospital cardiac arrest - App .

Resuscitation - Resuscitation-free interval . First responder system · Automated external defibrillators (AED)
Datenbank des FirstAED-Servers eingetragen. Die AED-Standorte sind in der Kartenansicht der App sichtbar. Alle Ersthelfer wurden aufgefordert, AED-Standorte, die noch nicht im System verzeichnet sind, zur Ergänzung der Datenbank $\mathrm{zu}$ melden.

\section{Ethik}

Das Forschungsvorhaben wurde von der Ethik-Kommission der Albert-LudwigsUniversität Freiburg genehmigt (Nr: 482/18) und beim Deutschen Register Klinischer Studien registriert $(\mathrm{Nr}$ : DRKS00016625).

\section{Datenerhebung}

Die Einsatzdaten werden im Backend des FirstAED-Servers gespeichert. Für die Ermittlung der Eintreffzeiten der Ersthelfer wurden die Ersthelfer im Einsatz geortet. Der Zeitpunkt, zu dem der Ersthelfer weniger als $100 \mathrm{~m}$ von der GPSPosition des Einsatzorts entfernt war, wurde als Eintreffzeit dokumentiert. Der Anteil der Einsätze, in denen mindestens 
Tab. 1 Ersthelfereinsätze im Projektzeitraum. Die Eintreffzeiten der Ersthelfer wurden mittels GPS-Position der Ersthelfer bestimmt, angegeben sind Mittelwert \pm Standardabweichung. In Klammern sind die absoluten Einsatzzahlen angegeben. Der Monat Juli 2018 wurde aufgrund des Testbetriebs ausgeschlossen

\begin{tabular}{|c|c|c|c|c|}
\hline Zeitraum & 2. Halbjahr 2018 & 1. Halbjahr 2019 & 2. Halbjahr 2019 & 1. Halbjahr 2020 \\
\hline Mittlere Helferzahl & 276 & 461 & 615 & 794 \\
\hline Anteil Einsatzübernahme & $30 \%(151 / 500)$ & $32 \%(204 / 631)$ & $34 \%(196 / 577)$ & $49 \%(164 / 334)$ \\
\hline Anteil Einsätze mit 1 Helfer & $58 \%$ & $68 \%$ & $62 \%$ & $51 \%$ \\
\hline Anteil Einsätze mit 2 Helfern & $28 \%$ & $20 \%$ & $25 \%$ & $24 \%$ \\
\hline Anteil Einsätze mit 3 Helfern & $11 \%$ & $8 \%$ & $10 \%$ & $12 \%$ \\
\hline Anteil Einsätze mit 4 Helfern & $3 \%$ & $4 \%$ & $3 \%$ & $13 \%$ \\
\hline Mittlere Eintreffzeit ersteintreffender Helfer (GPS) & $05: 18 \pm 03: 21(94)$ & $05: 45 \pm 04: 22(145)$ & $06: 09 \pm 04: 42(147)$ & $06: 09 \pm 03: 39(96)$ \\
\hline Mittlere Eintreffzeit ersteintreffendes Rettungsmittel & $07: 33 \pm 03: 37(483)$ & $07: 14 \pm 03: 39(624)$ & $07: 18 \pm 03: 25(566)$ & $07: 28 \pm 03: 24(322)$ \\
\hline $\begin{array}{l}\text { Eintreffen vor Rettungsdienst } \\
(\text { GPS })^{a, b}\end{array}$ & $28 \%(26 / 92)$ & $22 \%(31 / 143)$ & $23 \%(32 / 142)$ & $20 \%(19 / 93)$ \\
\hline Eintreffen vor Rettungsdienst (Helferbericht) ${ }^{\mathrm{a}}$ & - & - & $32 \%(31 / 98)$ & $44 \%(120 / 275)$ \\
\hline
\end{tabular}

ein Ersthelfer den Alarm akzeptiert hat, an der Gesamtheit der Einsätze, in denen das System FirstAED aktiviert wurde, bestimmt die Kennzahl Einsatzübernahme. Die deskriptive statistische Auswertung erfolgte mit Microsoft Excel.

Nach jedem Einsatz wurden die Ersthelfer über die App gebeten, einen Fragebogen auszufüllen. Es wurden auch Freitextantworten zur Zufriedenheit mit der App-Alarmierung bzw. zu Verbesserungsvorschlägen abgefragt. Diese wurden thematisch zusammengefasst und qualitativ ausgewertet.

\section{Studienablauf}

Das System wurde am 01.07.2018 gestartet, zeitgleich wurde mit der Helferrekrutierung begonnen. In der Zeit vom 01.07. bis 31.07.2018 erfolgte der Testbetrieb mit einer kleinen Anzahl von Helfern, die Software und Abläufe intensiv getestet haben. Alle Einsätze in der Zeit vom 01.08.2018 bis 30.06.2020 wurden in die Auswertung übernommen. In der Zeit vom 16.03.2020 bis 26.05.2020 wurde das System aufgrund der COVID-19Pandemie-bedingten Vorgaben für die Alarmierung von HvO-Systemen pausiert. Eine gesonderte Auswertung der Eintreffzeiten wurde vom 01.07 .2020 bis 31.07.2020 durchgeführt.
Optimierungen im Pilotbetrieb gemäß PDCA(plan-do-check-act)Zyklus

\section{Ergebnisse}

\section{Ersthelferzahlen}

Die Autoren evaluierten die Daten aus dem Pilotbetrieb alle sechs Monate. Nach jeder Halbjahresevaluation wurden Optimierungsmöglichkeiten eruiert und priorisiert. Hierbei arbeiteten die Autoren auch mit den Entwicklern der Firma FirstAED zusammen, um bei der Diskussion um softwareseitige Optimierungen Realisierbarkeit und Entwicklungsaufwand abschätzen zu können. Bei allen Optimierungen wurde nach dem PDCA-Zyklus vorgegangen. Neue Features oder Optimierungen wurden geplant und spezifiziert („plan“). Nach Programmierung wurde zunächst unter Laborbedingungen und anschließend von einer kleinen Gruppe Ersthelfer unter Realbedingungen getestet. Nach Korrektur eventueller Fehler wurde die Änderung für alle Benutzer freigegeben („do“). Die Verbesserungen des Systems durch die Optimierung der Software wurden anhand der Einsatzdaten im Echtbetrieb in den folgenden Monaten evaluiert („check“) und im Rahmen der Halbjahresevaluation erneut diskutiert. Daraufhin erfolgten bei Bedarf weitere Optimierungen (,act“).
Die Entwicklung der Anzahl registrierter Ersthelfer im System ist in - Tab. 1 aufgeführt. Im ersten Halbjahr 2020 wurden die Alarmierungen im Zeitraum vom 15.03. bis 26.05. aufgrund der Coronapandemie ausgesetzt. Die Registrierung neuer Ersthelfer wurde in dieser Zeit fortgeführt.

\section{Einsatzübernahmen}

In $\bullet$ Tab. 1 sind für die vier Halbjahre des Pilotprojekts die Gesamtzahl der Alarmierungen sowie die Anzahl der Einsätze, in denen mindestens ein Ersthelfer den Einsatz übernommen hat, aufgeführt. Die Steigerung der Ersthelferzahlen im 1. Halbjahr 2020 führte zum größten Anstieg der Einsatzübernahme (von 34 auf $49 \%$ ). In • Abb. 2 ist für die Postleitzahlenbereiche in der Projektregion die Helferdichte (Wohnadresse) farblich gekennzeichnet. Jeder Einsatz im Erhebungszeitraum ist mit einem Punkt markiert. In den Regionen mit geringerer Helferdichte werden nur selten Einsätze übernommen.

\section{Eintreffzeiten der Ersthelfer}

Die Eintreffzeiten der jeweils ersteintreffenden Ersthelfer sind in - Tab. 1 aufgeführt. Ebenso aufgeführt sind die 


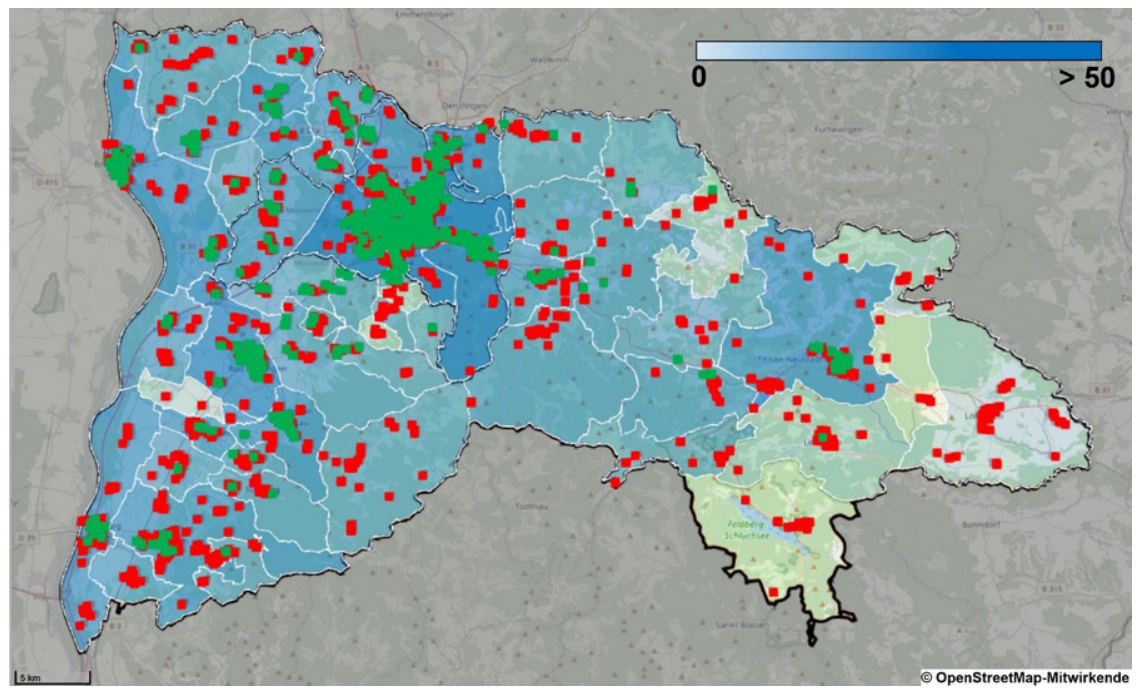

Abb. 2 A Die Karte zeigt die Einsatzorte für alle Einsätze im Zeitraum 01.01.2019 bis 30.06.2020. Alarme, bei denen Helfer den Einsatz übernommen haben, sind grün dargestellt. Alarme, bei denen keine Helfer den Einsatz akzeptiert haben, sind rot dargestellt. Die Helferdichte (Wohnort) in den Postleitzahlenbereichen ist blau dargestellt. (@ OpenStreetMap, Lizenz ODbL 1.0)

mittleren Eintreffzeiten des ersteintreffenden Rettungsmittels. Der Anteil der Einsätze, in denen ein Ersthelfer den Einsatzort vor dem Rettungsdienst erreichte, war im Pilotzeitraum nahezu unverändert (• Tab. 1).

\section{Verfügbarkeit von AED}

Die Abfrage bereits vorhandener AED im zweiten Halbjahr 2018 ergab eine Anzahl von 190 Geräten. 50 davon waren ohne Zutrittsbeschränkung verfügbar und konnten im System hinterlegt werden.

\section{Rückmeldungen der Ersthelfer über den Helferreport}

Die Fragen des Helferreports waren initial in der App hinterlegt und jedem Helfer wurden dieselben Fragen gestellt. So wurde beispielsweise gefragt, ob die Herzdruckmassage durchgeführt wurde, auch wenn der Helfer den Einsatzort nicht erreicht hatte. Deshalb wurde im 1. Halbjahr 2019 ein neuer Helferreport programmiert. Die Fragen sind in einer Datenbank hinterlegt, auf die aus der App zugegriffen wird. Dem Ersthelfer werden nur noch die für ihn relevanten Fragen präsentiert.

Nach Veröffentlichung des neuen Helferreports wurden im zweiten Halbjahr 2019 und im ersten Halbjahr 2020 insge- samt 167 Helferberichte mit ausgefülltem Freitextfeld abgeschickt. Diese wurden thematisch zusammengefasst. 7 Themen wurden wiederkehrend berichtet:

Einsätze verpasst. Das Smartphone war auf lautlos oder Vibration gestellt, bei der Alarmierung wurde kein Ton abgespielt. Manche Helfer berichteten, dass sie die Alarmierung verzögert wahrgenommen haben. Andere berichteten, dass sie auch schon Alarme verpasst haben und erst zu spät den verpassten Alarm entdeckt haben (Meldung im Sperrbildschirm des Smartphones) (28 Meldungen).

Strecke zum Notfallort nicht in angemessener Zeit zu bewältigen. Aufgrund zu großer Entfernung zum Einsatzort bzw. kurzer Eintreffzeit des Rettungsdiensts hat der Ersthelfer den Patienten nicht vor dem Rettungsdienst erreicht. Auch wurde bemängelt, dass das Verkehrsmittel des Ersthelfers nicht berücksichtigt wird (27 Meldungen).

\section{Verzögerung durch Abholen des AED.}

Der Ersthelfer hatte die Aufgabe, einen AED zur Einsatzstelle zu bringen. Ein anderer, mit AED ausgerüsteter Ersthelfer war im selben Einsatz, das Abholen des AED war somit unnötig (9 Meldungen).
Einsatzradius. Rückmeldungen von Ersthelfern, die bei sehr kurzer Eintreffzeit des Rettungsdiensts erst nach den Rettungsmitteln eingetroffen sind (5 Reports). Zusätzlich wurde beobachtet, dass im ländlichen Bereich nur selten Ersthelfer den Einsatz übernehmen. Wunsch nach größerem Alarmierungsradius bei langen Eintreffzeiten des Rettungsdiensts.

Zusätzlich kamen Rückmeldungen per E-Mail bezüglich der fehlenden Möglichkeit, sich als Ersthelfer am Notfallort auszuweisen. In Einzelfällen wurde Ersthelfern der Zutritt zur Wohnung des Patienten verweigert.

\section{Optimierungen des Systems während der Pilotphase}

\section{Dynamischer Alarmradius}

Ursprünglich wurden Ersthelfer im Umkreis von $1500 \mathrm{~m}$ um die Einsatzstelle alarmiert. Seit Juli 2019 übermittelt Rescuetrack bei jedem Einsatz die voraussichtliche Fahrtzeit („estimated time enroute " $[\mathrm{ETE}]$ ) von Rettungswagen und Notarzt auf der Basis von Verkehrslage und antizipierter Geschwindigkeit und schickt die kürzere ETE an den FirstAED-Server. In den Einstellungen des Systems wird festgelegt, für welche ETE welcher Alarmradius angewendet wird (• Abb. 3).

Die Konfiguration des Einsatzradius im Pilotprojekt ist in $\bullet$ Tab. 2 dargestellt. Zunächst wurde der Einsatzradius für voraussichtliche Fahrzeiten bis zu $5 \mathrm{~min}$ auf $1000 \mathrm{~m}$ festgelegt. Weiterhin wurde bei Fahrzeiten von über 12 min der Einsatzradius auf $3000 \mathrm{~m}$ erhöht.

Anfang 2020 wurde bei stark gestiegener Anzahl registrierter Ersthelfer der Einsatzradius für sehr kurze Eintreffzeiten des Rettungsdiensts weiter reduziert auf $600 \mathrm{~m}$. Dies führte nicht zu einer Reduktion der Einsatzübernahmen; die Einsatzübernahmen ließen sich - bei steigender Helferzahl - erhöhen.

\section{"Critical alert"}

Um die Wahrscheinlichkeit zu reduzieren, dass ein Ersthelfer eine Alarmierung nicht bemerkt, weil das Smartphone auf lautlos oder Vibrationsalarm gestellt ist, wurde für die Android- und iOS-Apps die 


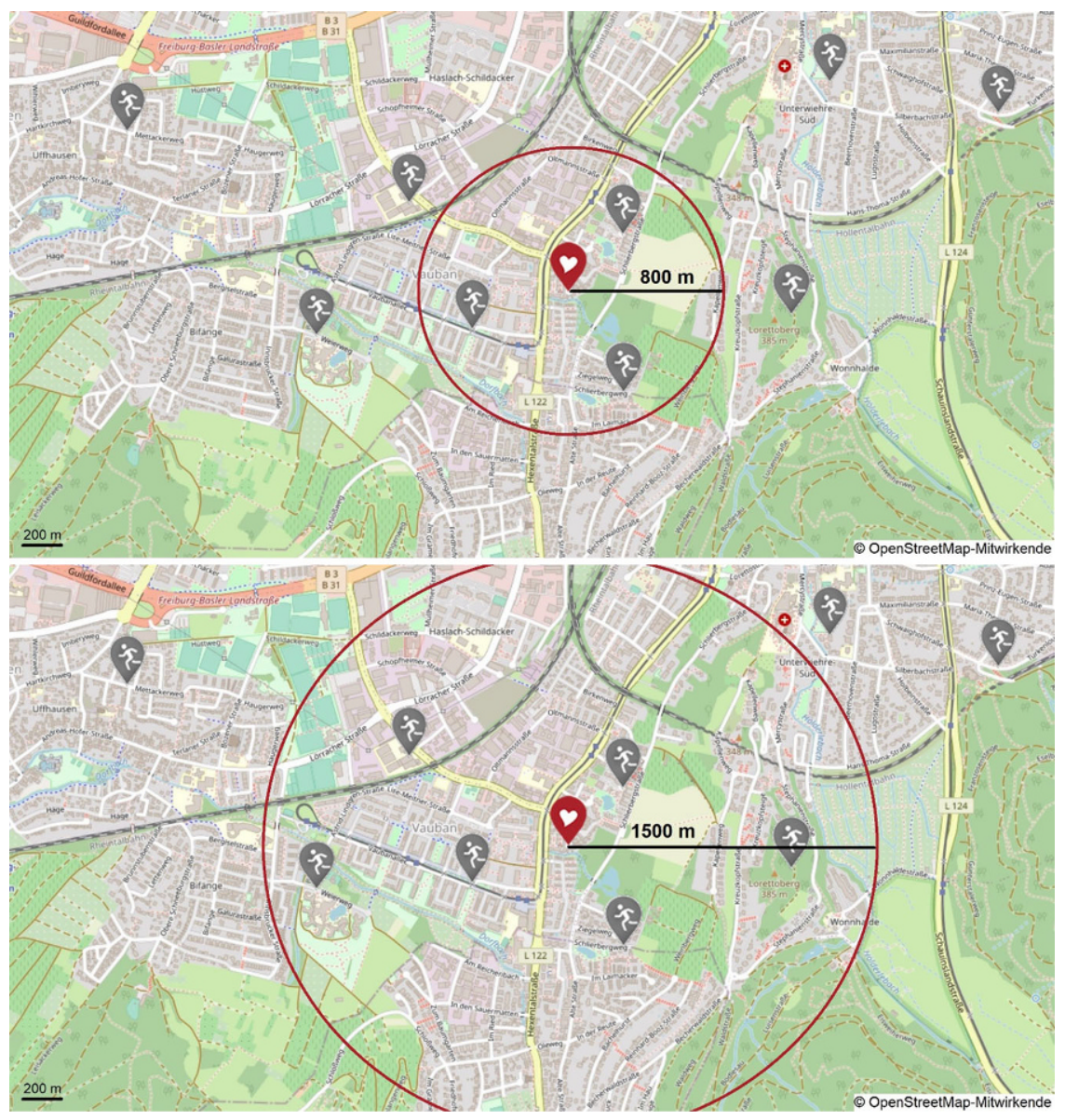

Abb. $3 \Delta$ Prinzip des dynamischen Alarmradius: Im oberen Bild sind die geschätzte Eintreffzeit des ersteintreffenden Rettungsmittels (ETE) bei 4 min und der Alarmradius klein. Im unteren Bild ist die ETE bei 10 min und der Alarmradius entsprechend größer. (๑ OpenStreetMap, Lizenz ODbL 1.0)

Möglichkeit entwickelt, dass das Smartphone trotz dieser Einstellung laut alarmiert. Für Android-Smartphones stand das neue Feature im zweiten Halbjahr 2019 zur Verfügung. Für die Criticalalert-Funktion in der iOS-App war ein Update der Serversoftware notwendig, das mit Umzug des Systems auf einen neuen Server im ersten Halbjahr 2020 verfügbar wurde.

\section{Verfügbarkeit von AED}

2020 wurde von FirstAED eine Schnittstelle zur AED-Datenbank DEFI-Map (www.defi-map.de) etabliert. Zusätzlich zum Standort können dadurch auch die Zeiten der Zutrittsbeschränkung eingetragen werden.

Die Besitzer von AED wurden kontaktiert und darum gebeten, den AED ohne Beschränkungen zugänglich zu machen. Auch wurde in den lokalen Medien dazu aufgerufen, öffentlich zugängliche AED fernung, Transportmittel und Eintreffzeit in Bezug zu setzen und mit diesen Daten den Algorithmus für Alarmradius und Auswahl der Ersthelfer bzw. Aufgabenverteilung zwischen den Ersthelfern zu optimieren.

\section{Eintreffzeiten}

Häufig war im System keine GPS-basierte Eintreffzeit dokumentiert, obwohl von Ersthelfern im Einsatzreport gemeldet wurde, dass diese die Einsatzstelle erreicht hatten. Um valide Daten für die Eintreffzeiten erheben zu können, wurde Ende Juni 2020 die Möglichkeit programmiert, die Statusmeldung „Ankunft beim Patienten“ innerhalb der App abzugeben. Im Juli wurden alle Einsätze hinsichtlich GPS-basierter Eintreffzeit und echter Eintreffzeit (= Statusmeldung) ausgewertet (mittlere Helferzahl: 870).

Bei insgesamt 129 Alarmierungen meldeten sich in 71 Fällen Ersthelfer (55\%). Insgesamt haben 130 Ersthelfer den Alarm akzeptiert. Über Statusmeldung wurde in 98 Fällen das Eintreffen dokumentiert. Die GPS-basierte Eintreffzeit wurde hingegen nur in 43 Fällen erfasst. In 32 Einsätzen wurde sowohl die GPS-basierte Eintreffzeit als auch die Statusmeldung dokumentiert. In 17 Fällen wurde das Eintreffen gemeldet, bevor die GPS-Position an der Einsatzstelle erfasst wurde (Abweichung -04:22 \pm 02:58 min $[\mathrm{MW} \pm \mathrm{SD}])$. In 15 Fällen wurde der Status „eingetroffen“ nach dem Erfassen der GPS-basierten Zeit dokumentiert (Abweichung +04:26 \pm 05:49 $\mathrm{min}$ $[\mathrm{MW} \pm \mathrm{SD}])$.

\section{Diskussion}

Im ersten Halbjahr 2020 wurde ein Ersthelferausweis für die App entwickelt. Bei Eintreffen an der Einsatzstelle zeigt dieser den Namen und ein Profilfoto des Ersthelfers sowie die Einsatznummer und den Hinweis, bei Unklarheiten die 112 anzurufen.

\section{Verkehrsmittel}

Anfang 2020 wurde eine Funktion entwickelt, bei der der Ersthelfer dem System unmittelbar nach Annahme eines Alarms über die App mitteilen muss, ob er zu Fuß, mit dem Fahrrad oder mit dem Pkw unterwegs ist. Damit ist es möglich, Ent-
Die Smartphone-basierte Ersthelferalarmierung stellt im Hinblick auf die Verkürzung reanimationsfreier Intervalle nur einen Teil eines zu optimierenden Systems dar. Durch Aktivieren von geschulten Ersthelfern in der Umgebung des Notfallorts können sehr kurze reanimationsfreie Intervalle resultieren. Ringh und Kollegen arbeiteten bereits vor fast 10 Jahren an einem System, welches Ersthelfer über Mobilfunkmasten orten und per Textnachricht (SMS) alarmieren kann [11]. Dieses System führte zu einer Reduktion des reanimationsfreien 
Tab. 2 Dynamischer Einsatzradius. In den ersten beiden Halbjahren war ein fester Alarmradius eingestellt. Für die beiden folgenden Halbjahre ist die Konfiguration des dynamischen Alarmradius angegeben. Das Rescuetrack-System übermittelt nach Alarmierung von Rettungswagen (RTW) und Notarzteinsatzfahrzeug (NEF) die voraussichtliche Fahrtzeit an den FirstAED-Server. Für die kürzere der beiden Zeiten wird der Alarmradius aus der Konfigurationstabelle übernommen

\begin{tabular}{|c|c|c|c|c|c|c|}
\hline & \multicolumn{6}{|c|}{ „Estimated time enroute“ (ETE) } \\
\hline & 0-3 min & 4-5 min & 6-9 min & $10-12 \mathrm{~min}$ & 13-17 min & $>17 \mathrm{~min}$ \\
\hline 2. Halbjahr 2018 & \multicolumn{6}{|c|}{1500 (fester Radius) } \\
\hline 1. Halbjahr 2019 & \multicolumn{6}{|c|}{1500 (fester Radius) } \\
\hline 2. Halbjahr 2019 & 1000 & 1000 & 1500 & 2000 & 2500 & 3000 \\
\hline 1. Halbjahr 2020 & 600 & 800 & 1000 & 1500 & 2500 & 3000 \\
\hline
\end{tabular}

Intervalls, allerdings ohne signifikanten Einfluss auf die Überlebensrate [12]. Im Schweizer Kanton Tessin wurde ein SMS-basiertes Alarmierungssystem für Ersthelfer mit der Alarmierung über eine Smartphone-App verglichen. Das Smartphone-basierte System führte zu einer Verkürzung der Eintreffzeiten und einer höheren Überlebensrate [2]. Mittlerweile sind verschiedene App-basierte Systeme etabliert und evaluiert [14]. Bisher besteht kein Konsens über die Indikation zum Ersthelfereinsatz. Erfolgt die Alarmierung nur bei Verdacht auf Herz-Kreislauf-Stillstand, so würde das System in der Region Freiburg/Breisgau Hochschwarzwald 200- bis 300-mal pro Jahr aktiviert werden. Bei 800 registrierten Helfern und 4 alarmierten Helfern pro Einsatz würde jeder Helfer im Schnitt 1-mal pro Jahr alarmiert werden. Das zusätzliche Einsatzstichwort „bewusstlos“ resultiert in einer etwa 5-fachen Einsatzhäufigkeit und schafft damit eine gewisse Routine in diesen Einsätzen. Weiterhin ist die Notrufmeldung oft ungenau und der Herz-Kreislauf-Stillstand wird als "bewusstlos" eingeordnet. Andere Ersthelferalarmierungssysteme werden auch bei weiteren Indikationen wie Atemnot oder Brustschmerz aktiviert [17]. Diese Einsatzindikationen wurden im vorliegenden Projekt ausgeschlossen, da ein Überlebensvorteil für die Patienten durch den Einsatz der Ersthelfer bei geringem Zeitvorteil und fehlender medizinischer Ausstattung der Helfer als unwahrscheinlich angenommen wurde und eine zu häufige Alarmierung die Motivation der Ersthelfer senken könnte.

Die Frage nach der benötigten Anzahl an Ersthelfern kann mit den vorliegenden Daten nicht beantwortet werden. Es ist fraglich, ob hier überhaupt Kennzahlen oder Empfehlungen erarbeitet werden können. Das Mobile-Retter-System in Gütersloh hatte bei einem Anteil registrierter Ersthelfer von 1,3 \%o der Bevölkerung eine Einsatzübernahme in $45 \%$ der Fälle [18]. Eine Steigerung der Ersthelferzahlen führte im hier beschriebenen Projekt zu einer Erhöhung der Einsatzübernahmen. Gerade in Regionen mit geringer Bevölkerungsdichte lässt sich die Anzahl der Ersthelfer jedoch nicht beliebig steigern. Hier kann das System mittels technologischer Innovationen verbessert werden. Die Autoren halten die Anpassung des Alarmierungsradius für Ersthelfer entsprechend der voraussichtlichen Fahrtzeit des Rettungsdiensts für essenziell. Durch die Reduzierung des Radius bei kurzen Fahrtzeiten des Rettungsdiensts profitiert das System: Es werden seltener Ersthelfer alarmiert, die erst nach dem Rettungsdienst eintreffen. Dies hat im Pilotprojekt zu einer deutlichen Steigerung der Motivation der Ersthelfer geführt. Weiterhin werden bei längeren Anfahrtszeiten Ersthelfer alarmiert, die zwar weiter vom Einsatzort entfernt sind, diesen allerdings immer noch vor dem Rettungsdienst erreichen können. Die Ergebnisse der vorliegenden Untersuchung zeigen auch, dass vor allem in den Randbereichen des Einsatzgebiets häufig kein Ersthelfer verfügbar war (•Abb. 2). Die Autoren vermuten, dass ein Anschluss der Nachbarkreise an das System zu einer höheren Helferdichte in der Grenzregion führen dürfte. Die leitstellengrenzen- bzw. landkreisgrenzenübergreifende Alarmierung von Ersthelfern könnte auch durch Schnittstellen der verschiedenen Systeme untereinander erreicht werden. Die Hersteller der Systeme sind hier aufgefordert, entsprechende Schnittstellen zu etablieren.
Im Projektzeitraum wurden die Ersthelferzahlen gesteigert und das System wurde zeitgleich technologisch weiterentwickelt. Dementsprechend lässt sich die Zunahme der Einsatzübernahmen nicht eindeutig der Implementierung des dynamischen Alarmradius einerseits oder den steigenden Ersthelferzahlen andererseits zuschreiben. Durch den dynamischen Alarmradius bedingt wurden bei Einsätzen mit voraussichtlich langen Eintreffzeiten Ersthelfer in wesentlich größerer Entfernung zum Einsatzort alarmiert. Dies könnte die etwas längeren Eintreffzeiten im 2. Halbjahr 2019 und im 1. Halbjahr 2020 erklären.

Im Pilotprojekt sollte auch die frühe Defibrillation vor Eintreffen des Rettungsdiensts realisiert werden. Deshalb wurde ein Softwaresystem ausgewählt, in welchem öffentlich zugängliche AED nicht nur sichtbar sind, sondern konkret einem Ersthelfer die Aufgabe zugeteilt wird, den AED zum Notfallort zu bringen. Werden die AED nur angezeigt, überlässt unter Umständen jeder Ersthelfer diese Aufgabe den anderen. In anderen Fällen bringen mehrere Ersthelfer einen AED zur Einsatzstelle. Das Abholen eines AED führte im System im Tessin zu einer Verzögerung von 97 (Anfahrt mit PKW) bzw. 555 s (fußläufige Ersthelfer; [1]). Im vorliegenden Projekt reichte die Dichte an öffentlich zugänglichen AED nicht aus. Dementsprechend wurde begonnen, neue AED strategisch zu positionieren. Der Verein Region der Lebensretter hat hierzu einen mit den Geräten im Rettungsdienst kompatiblen AED ausgewählt, so dass bei jedem Einsatz die Elektroden des AED durch den Rettungsdienst ersetzt werden, damit das Gerät sofort wieder einsatzbereit ist. Der Verein kauft die neuen Geräte, betreibt sie und kümmert sich um die Wartung im Sinne eines ganzheitlichen Ansatzes. Damithaben spendenbereite Bürger oder Firmen minimalen Aufwand. Eine genauere Untersuchung zur Verwendung der AED ist geplant.

Die Frage, ob die Ersthelferalarmierung über Smartphone die Überlebenswahrscheinlichkeit nach Herz-KreislaufStillstand erhöht, kann noch nicht beantwortet werden [14]. Reanimationsregister und Datenbanken wie beispiels- 
weise die Stelle für die Qualitätssicherung im Rettungsdienst (SQR-BW), in denen rettungsdienstliche Einsatzdaten erfasst werden, sollten zukünftig aufnehmen, ob ein Ersthelferalarmierungssystem aktiviert wurde, und die Einsatzzeiten (Alarmzeit, Eintreffzeit) erfassen. Voraussetzung hierfür ist die valide Datenerhebung. Dies ist jedoch zumindest in Deutschland bislang nicht etabliert. Für das Mobile-Retter-System in Gütersloh wurden sehr kurze Eintreffzeiten (Median: $4 \mathrm{~min}$ ) publiziert [17]. Die Methode zur Bestimmung der Eintreffzeiten wird nicht angegeben [9]. Die Autoren schreiben auf Nachfrage, dass eine automatisierte Erfassung der Eintreffzeiten erfolgte [7]. In einer anderen Arbeit geben dieselben Autoren an, der Ersthelfer würde als eingetroffen erfasst, sobald das Smartphone keine Ortsbewegung mehr erfasst [16]. In der aktuellsten Arbeit aus der Arbeitsgruppe wird letzten Endes erklärt, dass eine Smartphone-basierte Erfassung der Eintreffzeiten nicht zuverlässig zur Verfügung steht [15]. Diese Arbeit stellt die anhand einer GoogleMaps-Kalkulation geschätzten Eintreffzeiten der Mobilen Retter in 7 Regionen dar. Die tatsächlichen Eintreffzeiten dürften deutlich länger sein. In der vorliegenden Untersuchung konnte gezeigt werden, dass auch die Erfassung der Eintreffzeit über die GPS-Position nur unzuverlässig funktioniert. Dies liegt unter anderem auch daran, dass die verwendete Hardware (Smartphone der Ersthelfer) sehr uneinheitlich ist. Auch die Festlegung eines Radius um die Einsatzstelle, innerhalb dessen der Ersthelfer als „eingetroffen" im System erfasst wird, stellt eine unlösbare Herausforderung dar. Mit größerem Radius steigt die Zuverlässigkeit, mit der der Helfer als eingetroffen erfasst wird. Zeitgleich sinkt die Genauigkeit der Dokumentation, denn der Ersthelfer wird unter Umständen schon als eingetroffen erfasst, wenn er noch $100 \mathrm{~m}$ von dem Einsatzort entfernt ist. Bei kleinerem Radius steigt die Genauigkeit. Es kann jedoch vorkommen, dass der Helfer den Kreis nicht betritt (Patient befindet sich nicht im Kreis mit Mittelpunkt der Einsatzadresse) oder die Position durch das Smartphone nicht ausreichend genau ermittelt wird (z. B. in Gebäuden).
Im Freiburger System dokumentieren die Ersthelfer den Status S4 („eingetroffen“) beim ersten Patientenkontakt und nicht wie im Rettungsdienst üblich beim Eintreffen an der Postadresse. Dies bildet eine valide Datengrundlage für Qualitätssicherung und Forschung.

Das Mitwirken aller am Rettungsdienst beteiligten Institutionen (Hilfsorganisationen, ILS, Landkreis, Krankenhäuser, Kostenträger) und der Austausch zwischen Betreibern, Leitstelle sowie Softwareanbieter ist eine wichtige Voraussetzung, um eine erfolgreiche Implementierung und einen nachhaltigen Betrieb zu gewährleisten.

\section{Fazit für die Praxis}

- Smartphone-basierte Alarmierungssysteme bieten die Möglichkeit, das reanimationsfreie Intervall durch den Einsatz von in der Wiederbelebung geschulten Ersthelfern zu verkürzen.

- Entgegen der häufig kommunizierten Meinung handelt es sich bei den Alarmierungssystemen nicht ausschließlich um eine App, sondern um ein komplexes System, welches aus einem Server mit entsprechender Systemsoftware, den angebundenen Smartphones der Ersthelfer sowie der Ersthelfer-App besteht.

- Das System ist über Schnittstellen mit dem Einsatzleitsystem, ggf. noch mit einer AED-Datenbank und weiteren Systemen verbunden. Für das Erreichen bestmöglicher Ergebnisse sollte ein ganzheitlicher Ansatz mit optimaler Anpassung des Systems an die Anforderungen und regionalen Gegebenheiten bestehen.

- Das System muss in der Lage sein, valide Daten für Qualitätssicherung, Weiterentwicklung und Bearbeitung wissenschaftlicher Fragestellungen zu liefern.

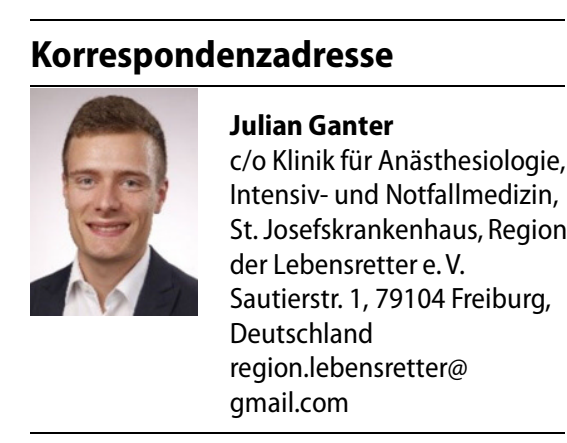

\section{Einhaltung ethischer Richtlinien}

Interessenkonflikt. G. Trummer, H.-J.Busch, K. Baldas, T. Steuber und M.P. Müller sind Vorstandsmitglieder im Verein Region der Lebensretter e. V. G. Trummer ist Vorstandsmitglied im Deutschen Rat für Wiederbelebung e. V. - German Resuscitation Council (GRC). H.-J. Busch und M.P. Müller sind Mitglied im Exekutivkomitee des GRC. J. Ganter, D. Damjanovic und J. Niechoj geben an, dass kein Interessenkonflikt besteht.

Für diesen Beitrag wurden von den Autoren keine Studien an Menschen oder Tieren durchgeführt. Für die aufgeführten Studien gelten die jeweils dort angegebenen ethischen Richtlinien.

\section{Literatur}

1. Auricchio A, Gianquintieri L, Burkart $R$ et al (2019) Real-life time and distance covered by lay first responders alerted by means of smartphone-application: Implications for early initiation of cardiopulmonary resuscitation and access to automatic external defibrillators. Resuscitation 141:182-187. https://doi.org/10 1016/j.resuscitation.2019.05.023

2. Caputo ML, Muschietti S, Burkart R et al (2017) Lay persons alerted by mobile application system initiate earlier cardio-pulmonary resuscitation: A comparison with SMS-based system notification. Resuscitation 114:73-78. https://doi.org/10.1016/ j.resuscitation.2017.03.003

3. Fischer Met al (2020) Jahresbericht des Deutschen Reanimationsregisters - Außerklinische Reanimation 2019. Anästh Intensivmed 61:V89-V93

4. Gräsner J-T, Geldner G, Werner C et al (2014) Optimierung der Reanimationsversorgung in Deutschland: Bad Boller Reanimationsgespräche 2014 - 10 Thesen für 10.000 Leben. Notfall Rettungsmed 17:314-316. https://doi.org/10. 1007/s10049-014-1879-y

5. Gross B, Schanderl F, Staedt N, Elsner C (2019) App-basierte Systeme zur Ersthelferalarmierung. Notfall Rettungsmed 22:483-491. https://doi.org/ 10.1007/s10049-018-0518-4

6. Henriksen FL, Schorling P, Hansen B et al (2016) FirstAED emergency dispatch, global positioning of community first responders with distinct roles - a solution to reduce the response times and ensuring an AED to early defibrillation in the rural area Langeland. Int J Netw Virtual Organ 16:86. https://doi.org/10.1504/IJNV0.2016.075131 
7. Hensel M, Stroop R, Strickmann B, Kerner T (2020) ReplytoletterbyMülleretal. Resuscitation.https:// doi.org/10.1016/j.resuscitation.2020.10.033

8. Kantar TNS (2018) D21-Digital-Index 2017/2018. Initiative D21 e 2018

9. Müller MP, Damjanovic D, Ganter J, Trummer G (2020) Comment on "Mobile phone-basedalerting of CPR-trained volunteers simultaneously with the ambulance can reduce the resuscitationfree interval and improve outcome after out-ofhospital cardiac arrest: A German, populationbased cohort study". Resuscitation. https://doi. org/10.1016/j.resuscitation.2020.09.041

10. Müller MP, Fischer M, Genzwürker H et al (2016) Smartphonebasierte Alarmierung von Ersthelfern bei der Reanimation: Es geht voran bei dieser wichtigen Maßnahme zur Reduktion des reanimationsfreien Intervalls. Notfall Rettungsmed 19:466-467. https://doi.org/10.1007/s10049016-0220-3

11. Ringh $M$, Fredman D, Nordberg $P$ et al (2011) Mobile phone technology identifies and recruits trained citizens to perform CPR on out-of-hospital cardiac arrest victims prior to ambulance arrival. Resuscitation 82:1514-1518. https://doi.org/10. 1016/j.resuscitation.2011.07.033

12. Ringh $M$, Rosenqvist $M$, Hollenberg J et al (2015) Mobile-phone dispatch of Laypersons for CPR in out-of-hospital cardiac arrest. N Engl J Med 372:2316-2325. https://doi.org/10.1056/ NEJMoa1406038

13. Sarkisian L, Mickley H, Schakow H et al (2020) Global positioning system alerted volunteer first responders arrive before emergency medical services in more than four out of five emergency calls. Resuscitation 152:170-176. https://doi.org/ 10.1016/j.resuscitation.2019.12.010

14. Scquizzato T, Pallanch O, Belletti A et al (2020) Enhancing citizens response to out-of-hospital cardiac arrest: a systematic review of mobilephone systems to alert citizens as first responders. Resuscitation 152:16-25.https://doi.org/10.1016/ j.resuscitation.2020.05.006

15. Stroop R, Hensel M, Kerner T (2020) Smartphonebasierte Ersthelferalarmierung - Auswertung der Alarmierungsdaten aus 7 Mobile-RetterRegionen. Der Notarzt 36(06):324-332. https:// doi.org/10.1055/a-1224-4103

16. Stroop R, Hensel M, Schnettker AT et al (2018) Smartphone-basierte Ersthelfer-Alarmierung verkürzt das reanima-tionsfreie Intervall. Eine Machbarkeitsstudie zur Verbesserung der präklinischen Reanimation. Anasth Intensivmed 59:58-67.https://doi.org/10.19224/ai2018.058

17. Stroop R, Kerner T, Strickmann B, Hensel M (2020) Mobile phone-based alerting of CPR-trained volunteers simultaneously with the ambulance can reduce the resuscitation-free interval and improve outcome after out-of-hospital cardiac arrest: a German, population-based cohort study. Resuscitation 147:57-64.https://doi.org/10.1016/ j.resuscitation.2019.12.012

18. Stroop R, Strickmann B, Horstkötter $\mathrm{H}$ et al (2015) Smartphone-basierte First-ResponderAlarmierung „Mobile Retter". Notarzt 31:239-245. https://doi.org/10.1055/s-0035-1552700

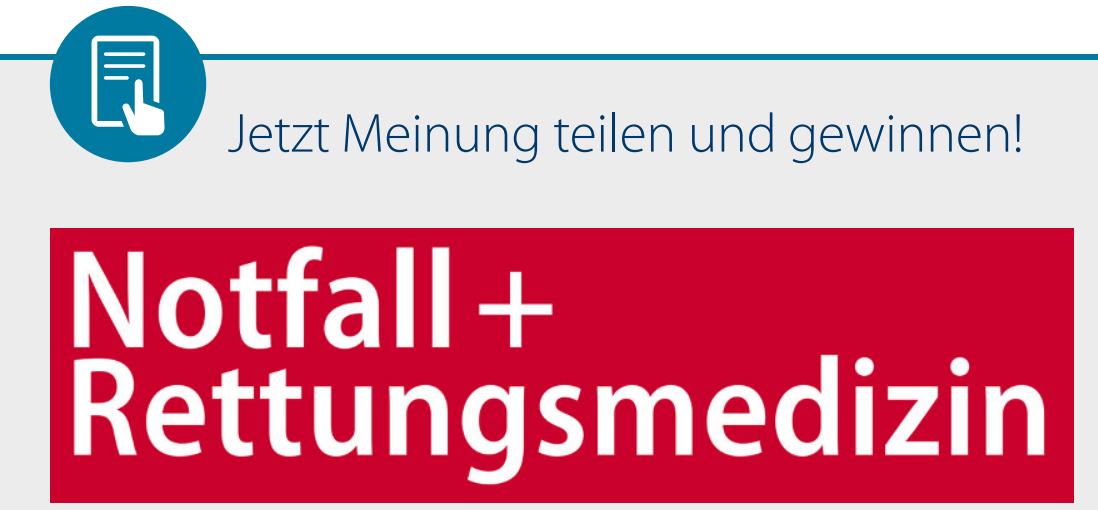

Liebe Leserinnen und Leser, in dieser Ausgabe feiern wir das 25-jährige Bestehen der Notfall+Rettungsmedizin. Wir haben dafür einige der langjährigen Herausgeber zu Wort kommen lassen - und nun sind Sie dran!

Wir laden Sie ein Ihre Sicht auf Notfall+Rettungsmedizin mit uns zu teilen. Und für 25 von Ihnen haben wir auch ein Geburtstagsgeschenk. Machen Sie mit!

Schicken Sie an ines.wolff@springer.com:

25 Worte zur Zeitschrift,

wie lange Sie die Zeitschrift schon lesen,

welcher Berufsgruppe Sie angehören,

und ein Foto von Ihnen (wenn Sie wollen).

\section{Gewinnen Sie!}

Es gibt 25 Gewinnpakete „Sauber retten!" zu gewinnen bestehend aus einem einzigartigen Kulturbeutel im Rot der Zeitschrift und dem dazu passenden Handtuch.

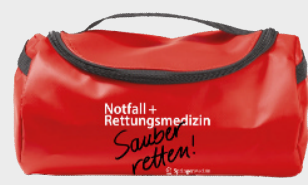

> Bitte geben Sie bei Ihrer Zusendung auch an, ob Sie einverstanden sind, dass Ihre Angaben gegebenfalls in einer Übersicht (wie auf Seit 172) in einer der kommenden Ausgabe erscheinen. Die Gewinnchance ist davon natürlich unabhängig. 